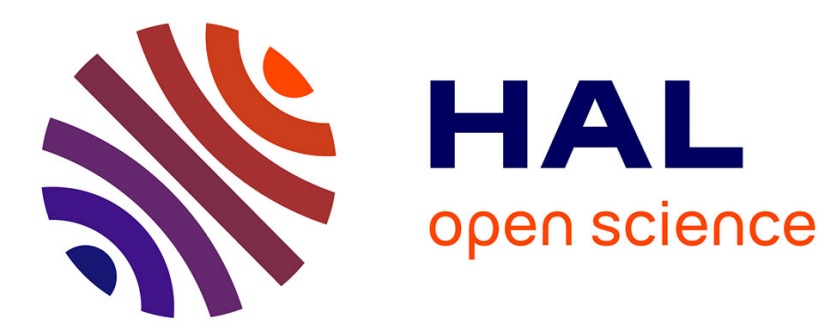

\title{
Consensus reaching in committees
}

\author{
Agnieszka Rusinowska, Patrik Eklund, Harrie de Swart
}

\section{To cite this version:}

Agnieszka Rusinowska, Patrik Eklund, Harrie de Swart. Consensus reaching in committees. European Journal of Operational Research, 2007, 178 (1), pp.185-193. halshs-00159838

\section{HAL Id: halshs-00159838 \\ https://shs.hal.science/halshs-00159838}

Submitted on 22 Jul 2009

HAL is a multi-disciplinary open access archive for the deposit and dissemination of scientific research documents, whether they are published or not. The documents may come from teaching and research institutions in France or abroad, or from public or private research centers.
L'archive ouverte pluridisciplinaire HAL, est destinée au dépôt et à la diffusion de documents scientifiques de niveau recherche, publiés ou non, émanant des établissements d'enseignement et de recherche français ou étrangers, des laboratoires publics ou privés. 


\title{
Consensus reaching in committees*
}

\author{
PATRIK EKLUND (1) \\ AGNIESZKA RUSINOWSKA ${ }^{\dagger}(2),(3)$ \\ HARRIE DE SWART (4)
}

(1) Umeå University, Department of Computing Science

SE-90187 Umeå, Sweden. E-mail: peklund@cs.umu.se

(2) Radboud University, Faculty of Management Science, P.O. Box 9108

6500 HK Nijmegen, The Netherlands. E-mail: a.rusinowska@fm.ru.nl

(3) Warsaw School of Economics, Department of Mathematical Economics

Al. Niepodleglosci 162, 02-554 Warsaw, Poland. E-mail: arusin@sgh.waw.pl

(4) Tilburg University, Faculty of Philosophy, P.O. Box 90153,

5000 LE Tilburg, The Netherlands. E-mail: H.C.M.deSwart@uvt.nl

\begin{abstract}
In this paper, we apply a consensus model to decision-making in committees that have to choose one or more alternatives from a set of alternatives. The model does not use a voting rule nor a set of winning coalitions. Every decision maker evaluates each alternative with respect to given criteria. The criteria may be of unequal importance to a decision maker. Decision makers may be advised by a chairman to adjust their preferences, i.e., to change their evaluation of some alternative(s) or/and the importance of the criteria, in order to obtain a better consensus. The consensus result should satisfy constraints concerning the consensus degree and the majority degree. A simple example is presented.
\end{abstract}

KEYWORDS: constraints satisfaction, consensus, committees

JEL CLASSIFICATION: C6

CORRESPONDING AUTHOR: Harrie de Swart

Tilburg University, Department of Philosophy, P.O. Box 90153, 5000 LE Tilburg, The Netherlands; E-mail: H.C.M.deSwart@uvt.nl

Tel.: 0031 - 13 - 46624 15; Fax: 0031 - 13 - 4662892

\footnotetext{
*The authors wish to thank Professor Hannu Nurmi for very useful discussions concerning consensus theory. They are also grateful to two anonymous referees for their suggestions.

${ }^{\dagger}$ This author gratefully acknowledges support by COST Action 274, TARSKI.
} 


\section{Introduction}

Usually, each member in a committee ranks the candidates or alternatives in a linear ordering and next some voting rule, like Plurality, Majority or Borda, is used to aggregate the individual rankings into a social ranking. It is well known that the outcome determined this way is very much dependent on the chosen aggregation procedure. Depending on the criterium used, also the individual rankings of the candidates may vary. In Nurmi (2005), the author gives a nice overview of the aggregation problems in policy evaluation.

These problems are also discussed in Peleg (1984), in which the author gives a game-theoretic analysis of voting in committees. A committee is defined as a game $G=(N, W)$, where $N$ is the set of committee members and $W$ is the set of winning coalitions. Given a profile $R^{N}$, alternative $x$ dominates $y$ if $\left\{i \in N \mid x R^{i} y\right\}$ is winning. Given a profile, the core is the set of undominated alternatives. The author shows that for a committee $G=(N, W)$ without veto players all cores are non-empty if and only if the number of alternatives is smaller than the Nakamura number of $G$.

In Laruelle and Valenciano (2005a, 2005b), the authors consider committees that bargain in search of consensus over a set of feasible agreements under a voting rule. They specify strict probabilistic bargaining protocols as well as protocols derived from the voting rules and investigate the stationary subgame perfect equilibria.

The approach in the present paper is completely different. First of all, the outcome is not determined by a voting rule nor by a set of winning coalitions. Instead, the present consensus model uses concepts introduced in Carlsson et al. (1992). Every decision maker evaluates each alternative with respect to given criteria, agreed upon in advance. Not only are the alternatives ranked by each member in a weak order, but also - more precisely and more detailed - each decision maker $i$ values the different alternatives $a$ with respect to each criterium $c$ by a real number $g_{i}(c, a)$ such that the sum of $g_{i}(c, a)$ over all alternatives $a$ equals to 1 . The different criteria may be of unequal weight to a decision maker. Let $h_{i}(c)$ be the weight that committee member $i$ assigns to criterium $c$, then we assume that the sum of $h_{i}(c)$ over all criteria $c$ equals to 1 . Based on the evaluations (comparisons) of all alternatives by all decision makers (taking into account all criteria and their weights for the different committee members), the Euclidean-like 'distances' between decision makers are calculated. The generalized consensus degree is defined as 1 minus the maximum distance between two members. In the model a minimum consensus degree is required in advance. If the computed generalized consensus degree is smaller than the required one, a procedure of consensus reaching starts in which the chairman asks at least one decision maker to adjust his preferences.

The model presented in this paper can be explained to a general audience much easier than the ones mentioned above. Consequently, it is relatively easy to use this model in applications, in particular since appropriate software for supporting the decision making is available. The present model is also closer to practice, since a special role is foreseen for the chairman of the committee. Fi- 
nally, since there is no voting rule involved, the aggregation problems mentioned above cannot occur.

Section 2 of this paper concerns the formal consensus model. In Subsection 2.1, we introduce the model, while in Subsection 2.2, we compare it with some other methods proposed in the literature. In Section 3, we apply the consensus model to the Management Committee of an EU Cost Action. Finally, in Section 4 , we conclude.

We do not address here the problem how to elect a committee or how to form a stable coalition. This problem is addressed among others in Brams et al. $(2003,2004)$.

\section{The consensus model}

\subsection{Description of the model}

The formal model is as follows. Let $N$ be the set of all decision makers who try to reach consensus on some alternatives. Let $A$ denote the set of all alternatives. Let $C$ denote the set of all criteria. Each decision maker is supposed to have an evaluation of the importance of the criteria. Hence, for each $i \in N$, we assume $h_{i}: C \rightarrow[0,1]$, such that

$$
\forall i \in N\left[\sum_{c \in C} h_{i}(c)=1\right],
$$

where $h_{i}(c)$ is $i$ 's evaluation (or weight) of criterion $c$. Moreover, for each $i \in N$, we also assume $g_{i}: C \times A \rightarrow[0,1]$ such that

$$
\forall c \in C\left[\sum_{a \in A} g_{i}(c, a)=1\right],
$$

where $g_{i}(c, a)$ is the value of alternative $a$ to decision maker $i$ with respect to criterion $c$.

In order to determine his evaluations $h_{i}(c)$ of the importance of the different criteria $c$ and his values $g_{i}(c, a)$ of the different alternatives $a$ with respect to a given criterion $c$, decision maker $i$ may use the MacBeth software (Bana e Costa and Vansnick, 1999; Bana e Costa et al., 2003), which is extremely appropriate for these purposes. The reasons for preferring the MacBeth method to Saaty's Analytical Hierarchy Process (Saaty 1977, 1980) are explained in Bana e Costa and Vansnick (2001); see also Section 4 for a discussion.

Let $\left(h_{i}(c)\right)_{c \in C}$ denote the $1 \times|C|$ matrix representing the evaluation (comparison) of the criteria by decision maker $i$, and let $\left(g_{i}(c, a)\right)_{c \in C, a \in A}$ denote the $|C| \times|A|$ matrix containing $i$ 's evaluation (comparison) of all alternatives with respect to each criterion in $C$. For each $i \in N$, we define $f_{i}: A \rightarrow[0,1]$ such that

$$
\left(f_{i}(a)\right)_{a \in A}=\left(h_{i}(c)\right)_{c \in C} \cdot\left(g_{i}(c, a)\right)_{c \in C, a \in A},
$$

where $f_{i}(a)$ is $i$ 's evaluation of alternative $a$, and $\left(f_{i}(a)\right)_{a \in A}$ is the $1 \times|A|$ matrix containing $i$ 's evaluation of each alternative. 
The Euclidean-like 'distances' between decision makers $i$ and $j$ are calculated as follows:

$$
d\left(f_{i}, f_{j}\right)=\sqrt{\frac{1}{|A|} \sum_{a \in A}\left(f_{i}(a)-f_{j}(a)\right)^{2}} .
$$

By $(1),(2)$, and $(3), 0 \leq d\left(f_{i}, f_{j}\right) \leq 1$. Let

$$
d^{*}=\max \left\{d\left(f_{i}, f_{j}\right) \mid i, j \in N\right\} .
$$

A generalized consensus degree $\delta^{*}$ for a committee is defined as

$$
\delta^{*}=1-d^{*}=1-\max \left\{d\left(f_{i}, f_{j}\right) \mid i, j \in N\right\} .
$$

In the model a certain consensus degree $\widetilde{\delta} \in(0,1]$ is required in advance. We say that a committee reaches consensus if the constraint $\delta^{*} \geq \widetilde{\delta}$ is satisfied.

In the likely situation that at the first stage no consensus is reached, committee members are tentatively partitioned according to their rankings of the alternatives. Each group of this partition is given time to explain and defend its position. Also, each member may persuade other members to adopt his point of view. If consequently some members change their mind, the new consensus degree is calculated. If this is still too small, i.e., less than $\widetilde{\delta}$, the process of consensus reaching should be continued. That is why we assume a kind of mediator, called the chairman. If the decision makers reach a consensus degree $\delta^{*}<\widetilde{\delta}$, it is the chairman who decides who should adjust his/her preferences in order to reach a better consensus. He does so in the following way. Let

$$
D^{*}=\left\{i \in N \mid \exists j \in N\left[d\left(f_{i}, f_{j}\right)=d^{*}\right]\right\} .
$$

The chairman selects a decision maker $i^{*} \in D^{*}$ to adjust his/her preferences, who satisfies the following condition

$$
i^{*}=\arg \min _{k \in D^{*}} d_{-k}^{*}
$$

where $d_{-k}^{*}$ is defined for $k \in D^{*}$ as

$$
d_{-k}^{*}=\max \left\{d\left(f_{i}, f_{j}\right) \mid i, j \in N \backslash\{k\}\right\} .
$$

If there are two members satisfying condition (8), the chairman chooses one of them. The chairman may also give advice to $i^{*}$ how to adapt his/her preferences. The chairman's advice should always lead to an increase of $\delta^{*}$. If $i^{*}$ refuses to change his/her preferences, he/she is 'excluded' from further discussion.

We assume a majority degree $\widetilde{m}$, that is, the minimal number of decision makers necessary to make a decision. If $\left|N \backslash\left\{i^{*}\right\}\right| \geq \widetilde{m}$, the remaining decision makers try to reach consensus. If $\left|N \backslash\left\{i^{*}\right\}\right|<\widetilde{m}$, the committee does not reach consensus. If $i^{*}$ follows the chairman's advice, then the new generalized consensus degree $\delta^{\prime *}$ is calculated, and if $\delta^{\prime *} \geq \widetilde{\delta}$, the committee reaches consensus. If $\delta^{*}<\widetilde{\delta}$, then a new decision maker, $i^{\prime *}$, is appointed by the chairman for adjusting his/her preferences, etc. 
If consensus is reached by the committee, that is, if the constraints concerning the consensus degree and the majority degree are satisfied, a mean consensus decision is calculated. Let $N^{*} \subseteq N$ denote the set of the decision makers who succeeded in reaching consensus. Assuming that the decision makers might be unequally 'important', we add up the weighted (final) values of the alternatives to all decision makers from $N^{*}$. For each $a \in A$, the weighted value $f(a)$ of alternative $a$ is defined as

$$
f(a)=\sum_{i \in N^{*}} w_{i}^{\prime} \cdot f_{i}(a)
$$

where for each $i \in N^{*}$

$$
w_{i}^{\prime}=\frac{w_{i}}{\sum_{j \in N^{*}} w_{j}},
$$

and $w_{i}$ means the 'weight' of decision maker $i \in N^{*}$. The committee chooses the alternatives with the greatest value of $f(a)$. In particular, if only one alternative may be chosen, the committee chooses the alternative $a^{*}$ such that

$$
a^{*}=\arg \max _{a \in A} f(a)
$$

If there are (at least) two alternatives satisfying condition (12), and only one alternative may be chosen, the chairman decides for one of them.

\subsection{Comparing the model with other methods}

In the literature, many procedures of choosing one or more alternatives from a set of available alternatives have been analyzed. In Section 1, we have already mentioned game-theoretical treatises like the ones by Peleg (1984), and Laruelle and Valenciano (2005a, 2005b).

In social choice theory, given available alternatives and individual preference orderings of the alternatives, an election mechanism can serve to select one alternative or, if needed, a set of alternatives. An overview and comparison of several election procedures is presented, for instance, in Brams and Fishburn (2002), and in de Swart et al. (2003). The most well-known election procedures are Plurality Rule (Most Votes Count), Majority Rule (Pairwise Comparison), Borda Rule, and Approval Voting.

Under the Plurality Rule (Most Votes Count), only the first preference of a voter is considered. An alternative $x$ is collectively preferred to an alternative $y$ if the number of voters that prefer $x$ most is greater than the number of voters that prefer $y$ most. Under the Plurality Rule, if one alternative must be chosen, it will be the alternative which is put first by most voters.

The Majority Rule (Pairwise Comparison) is based on the majority principle which says that alternative $x$ is collectively preferred to $y$ if $x$ defeats $y$, i.e., the number of voters that prefer $x$ to $y$ is greater than the number of voters that prefer $y$ to $x$. If there is an alternative that defeats every other alternative in pairwise comparison, this alternative must be chosen and it is called a Condorcet winner (Condorcet, 1788). 
Under the Borda Rule (Borda, 1781), weights are given to all the positions of the alternatives in the individual preferences. For $n$ alternatives, every voter gives $n$ points to his most preferred alternative, $n-1$ points to his second alternative, etc., and 1 point to his least preferred alternative. A decision is made based on the total score of every alternative in a given voter profile.

Under Approval Voting (Brams and Fishburn, 1983), each voter divides the alternatives into two classes: the alternatives he approves of and the ones he disapproves of. Each time an alternative is approved of by a voter is good for one point. The alternative chosen is the one that receives most points.

As becomes clear from Subsection 2.1, we propose a completely different procedure. The point of departure for our model is a method proposed in Carlsson et al. (1992), where the authors study the problem of formalizing consensus, within a set of decision makers trying to agree on a mutual decision. Convergence to consensus depends on the willingness of the decision makers to compromise. In the present paper, we formulate a consensus model for decision-making in committees. Each decision maker evaluates each alternative with respect to certain criteria, agreed upon in advance. Based on the evaluations of all alternatives by all decision makers, the 'distances' between decision makers, and next a generalized consensus degree, are calculated. If the generalized consensus degree is smaller than a certain required one, the procedure of consensus reaching starts in which at least one decision maker is asked to adjust his preferences.

The consensus model presented in this paper is much richer and more precise than the election mechanisms from social choice theory. The profiles consisting of the individual rankings of the alternatives are a by-product of our more detailed and more sophisticated evaluations $f_{i}(a)$ of the different alternatives $a$ by individual $i$. For each $i$ one can derive the ranking of the alternatives by individual $i$ from the evaluations $f_{i}(a)$, but not conversely. In going from the evaluations to the individual ordering a lot of information is lost. The evaluations are with respect to given criteria, which may have different weights. The evaluations $f_{i}(a)$ are based on the weights $h_{i}(c)$ of the given criteria $c$ and on the values $g_{i}(c, a)$ of alternative $a$ to decision maker $i$ with respect to the different criteria $c$. In order to determine the weights $h_{i}(c)$ and the values $g_{i}(c, a)$ one may use the MacBeth software, which is very user-friendly. Since so much information is lost when going from the evaluations $f_{i}(a)$ to the individual orderings of the alternatives it is no surprise that different election mechanisms applied to these individual rankings give different outcomes.

Another added value of our model is that, contrary to the other 'static' methods, it is dynamic, giving voters a chance to adjust their preferences. There is a lot of room for negotiations here which makes our model very appropriate for practical applications. It seems much harder to apply the game theoretic approaches mentioned earlier to practical situations.

As mentioned before, our model is different from the methods proposed in the literature, in particular, from the approach presented in Peleg (1984). In particular, our consensus outcome is not determined by a set of winning coalitions. Nevertheless, one should notice that given a (sub)set of decision makers 
who manage to reach consensus, and given their final (adjusted) preferences, the consensus outcome is undominated in the sense that there is no alternative which is strongly preferred to the consensus outcome by all decision makers in question.

\section{The example}

In this section, we present an application of the consensus model to an EU COST Action activity. Suppose that the Management Committee (MC) of the COST Action has to choose candidates for a grant for a 'Short Term Scientific Mission' (STSM). We consider a small MC, consisting of only five members, i.e.,

$$
N=\{1,2,3,4,5\}
$$

Suppose there are two grants to be assigned, but four applicants, i.e.,

$$
A=\left\{a_{1}, a_{2}, a_{3}, a_{4}\right\}
$$

Each MC member will evaluate each applicant with respect to three criteria. We have

$$
C=\left\{c_{1}, c_{2}, c_{3}\right\}
$$

where

$c_{1}$ is the quality of the detailed work plan,

$c_{2}$ is the relevance of the STSM proposal to the COST Action and to the research conducted by the host group, and

$c_{3}$ is the quality of the $\mathrm{CV}$ of the applicant.

The majority degree is assumed to be $\widetilde{m}=3$, i.e., at least three out of five MC members may make a decision. Since the STSM grants are very attractive to junior researchers, the MC wants to be rather unanimous in choosing the candidates, and the required consensus degree is $\widetilde{\delta}=0.95$. If more than two applicants are chosen by our consensus method, then we propose the chairman to choose only two of them.

The relative weights that the different $\mathrm{MC}$ members attach to the criteria $c_{1}, c_{2}, c_{3}$ are as follows:

$$
\begin{gathered}
h_{1}=(0.4,0.3,0.3), \quad h_{2}=(0.4,0.4,0.2), \quad h_{3}=(0.1,0.1,0.8), \\
h_{4}=(0.4,0.2,0.4), \quad h_{5}=(0.2,0.6,0.2) .
\end{gathered}
$$

MC member 3, for instance, seems to be the most 'extreme' in his opinion about the importance of the criteria: when evaluating the applicants, he focuses mainly on the applicant's CV, and does not pay too much attention to the work plan nor to the relevance of the proposal to the Cost Action and to the research of the host group. In decision maker 5's opinion, the latter is the most important criterion when evaluating the applicants, etc. 
Moreover, we assume that

$$
\begin{aligned}
\left(g_{1}(c, a)\right)_{c \in C, a \in A} & =\left(\begin{array}{cccc}
0.2 & 0.3 & 0.3 & 0.2 \\
0.1 & 0.2 & 0.4 & 0.3 \\
0.5 & 0.2 & 0.2 & 0.1
\end{array}\right) \\
\left(g_{2}(c, a)\right)_{c \in C, a \in A} & =\left(\begin{array}{cccc}
0.35 & 0.25 & 0.2 & 0.2 \\
0.25 & 0.25 & 0.25 & 0.25 \\
0.2 & 0.5 & 0.2 & 0.1
\end{array}\right) \\
\left(g_{3}(c, a)\right)_{c \in C, a \in A} & =\left(\begin{array}{cccc}
0.3 & 0.4 & 0.1 & 0.2 \\
0.2 & 0.1 & 0.2 & 0.5 \\
0.3 & 0.4 & 0.2 & 0.1
\end{array}\right) \\
\left(g_{4}(c, a)\right)_{c \in C, a \in A} & =\left(\begin{array}{cccc}
0.25 & 0.25 & 0.25 & 0.25 \\
0.1 & 0.2 & 0.5 & 0.2 \\
0.25 & 0.35 & 0.2 & 0.2
\end{array}\right) \\
\left(g_{5}(c, a)\right)_{c \in C, a \in A} & =\left(\begin{array}{cccc}
0.3 & 0.2 & 0.3 & 0.2 \\
0.5 & 0.1 & 0.2 & 0.2 \\
0.5 & 0.2 & 0.1 & 0.2
\end{array}\right) .
\end{aligned}
$$

Hence, by virtue of (3), the MC members' evaluations of the applicants are as follows:

$$
\begin{gathered}
\left(f_{1}(a)\right)_{a \in A}=(0.26,0.24,0.3,0.2), \quad\left(f_{2}(a)\right)_{a \in A}=(0.28,0.3,0.22,0.2), \\
\left(f_{3}(a)\right)_{a \in A}=(0.29,0.37,0.19,0.15), \quad\left(f_{4}(a)\right)_{a \in A}=(0.22,0.28,0.28,0.22), \\
\left(f_{5}(a)\right)_{a \in A}=(0.46,0.14,0.2,0.2) .
\end{gathered}
$$

Table 1 presents the distances between the MC members.

Table 1: Distances $d\left(f_{i}, f_{j}\right)$, where $i, j \in N=\{1,2,3,4,5\}$.

\begin{tabular}{c|c|c|c|c|c} 
& 1 & 2 & 3 & 4 & 5 \\
\hline 1 & & 0.051 & 0.09 & 0.0316 & 0.1225 \\
\hline 2 & 0.051 & & 0.0458 & 0.0447 & 0.1208 \\
\hline 3 & 0.09 & 0.0458 & & 0.0806 & 0.1453 \\
\hline 4 & 0.0316 & 0.0447 & 0.0806 & & 0.1449 \\
\hline 5 & 0.1225 & 0.1208 & 0.1453 & 0.1449 & \\
\hline
\end{tabular}

Hence, we get

$$
d^{*}=d\left(f_{3}, f_{5}\right)=0.1453, \quad D^{*}=\{3,5\} .
$$

One of the MC members from $D^{*}$ will be asked to change his/her preferences. In order to find out which one, we calculate $d^{*}$ when decision maker 3 is removed, and $d^{*}$ when decision maker 5 is removed. Table 2 presents the distances between the MC members when 3 is removed, and Table 3 presents the distances when 5 is removed. 
Table 2: Distances $d\left(f_{i}, f_{j}\right)$, where $i, j \in N=\{1,2,4,5\}$.

\begin{tabular}{c|c|c|c|c} 
& 1 & 2 & 4 & 5 \\
\hline 1 & & 0.051 & 0.0316 & 0.1225 \\
\hline 2 & 0.051 & & 0.0447 & 0.1208 \\
\hline 4 & 0.0316 & 0.0447 & & 0.1449 \\
\hline 5 & 0.1225 & 0.1208 & 0.1449 & \\
\hline
\end{tabular}

Table 3: Distances $d\left(f_{i}, f_{j}\right)$, where $i, j \in N=\{1,2,3,4\}$.

\begin{tabular}{c|c|c|c|c} 
& 1 & 2 & 3 & 4 \\
\hline 1 & & 0.051 & 0.09 & 0.0316 \\
\hline 2 & 0.051 & & 0.0458 & 0.0447 \\
\hline 3 & 0.09 & 0.0458 & & 0.0806 \\
\hline 4 & 0.0316 & 0.0447 & 0.0806 & \\
\hline
\end{tabular}

Hence,

$$
d_{-3}^{*}=d\left(f_{4}, f_{5}\right)=0.1449, \quad d_{-5}^{*}=d\left(f_{1}, f_{3}\right)=0.09 .
$$

Therefore, it is decision maker 5 who is asked to change his preferences, i.e.,

$$
i^{*}=5 .
$$

Suppose that decision maker 5 announces that he will not change his preferences at all, and hence, the MC decides to exclude him from further discussion. Now, only decision makers $1,2,3$, and 4 try to reach consensus. We have then

$$
d^{\prime *}=0.09, \quad D^{\prime *}=\{1,3\},
$$

and moreover,

$$
d_{-1}^{\prime *}=0.0806, \quad d_{-3}^{\prime *}=0.051,
$$

which gives

$$
i^{\prime *}=3 .
$$

Hence, decision maker 3 is asked to change his preferences. Let us suppose that he is very cooperative, and agrees to follow the chairman's advice. Assume his new preferences are as follows:

$$
\begin{gathered}
h_{3}^{\prime}=(0.3,0.3,0.4) \\
\left(g_{3}^{\prime}(c, a)\right)_{c \in C, a \in A}=\left(\begin{array}{cccc}
0.3 & 0.3 & 0.2 & 0.2 \\
0.2 & 0.1 & 0.3 & 0.4 \\
0.3 & 0.4 & 0.2 & 0.1
\end{array}\right)
\end{gathered}
$$

and consequently, decision maker 3's new evaluations are

$$
\left(f_{3}^{\prime}(a)\right)_{a \in A}=(0.27,0.28,0.23,0.22) .
$$

Table 4 shows the distances between decision makers from the set $\{1,2,3,4\}$ after 3 adjusted his preferences. 
Table 4: Distances $d\left(f_{i}^{\prime}, f_{j}^{\prime}\right)$, where $i, j \in\{1,2,3,4\}$, and $f_{i}^{\prime}=f_{i}$ for $i \in\{1,2,4\}$.

\begin{tabular}{c|c|c|c|c} 
& 1 & 2 & 3 & 4 \\
\hline 1 & & 0.051 & 0.0418 & 0.0316 \\
\hline 2 & 0.051 & & 0.0158 & 0.0447 \\
\hline 3 & 0.0418 & 0.0158 & & 0.0354 \\
\hline 4 & 0.0316 & 0.0447 & 0.0354 & \\
\hline
\end{tabular}

We have then

$$
d^{\prime \prime *}=0.051, \quad D^{\prime \prime *}=\{1,2\}, \quad d_{-1}^{\prime \prime *}=0.0447, \quad d_{-2}^{\prime \prime *}=0.0418,
$$

and hence decision maker 2 is asked to change his preferences, i.e.,

$$
i^{\prime \prime *}=2 .
$$

Decision maker 2 would be willing, in principle, to adjust his preferences. Nevertheless, since he can see that even without him a decision will be made, he refuses to change his preferences. In this situation, that is, with decision maker 2 removed, we have

$$
m=3 \geq \widetilde{m}, \quad d^{\prime \prime \prime *}=0.0418, \quad \delta^{\prime \prime \prime *}=0.9582>0.95=\widetilde{\delta},
$$

and therefore $\mathrm{MC}$ members 1,3 , and 4 reach consensus. In order to choose two candidates, they calculate the total values of the candidates. The MC decides to treat all the members equally, that is, for $k=1,2,3,4$ we have

$$
f\left(a_{k}\right)=\frac{1}{3} f_{1}\left(a_{k}\right)+\frac{1}{3} f_{3}^{\prime}\left(a_{k}\right)+\frac{1}{3} f_{4}\left(a_{k}\right) .
$$

Hence,

$$
f\left(a_{1}\right)=\frac{0.75}{3}, \quad f\left(a_{2}\right)=\frac{0.8}{3}, \quad f\left(a_{3}\right)=\frac{0.81}{3}, \quad f\left(a_{4}\right)=\frac{0.64}{3}
$$

and choosing two candidates with the greatest total value, the MC members propose to give the STSM grants to candidates $a_{2}$ and $a_{3}$.

Next, let us see what the outcome would be if we apply different voting rules

\begin{tabular}{|c|c|c|}
\hline 1: & $a_{3}$ & $a_{2}$ \\
\hline 2: & $a_{2}$ & $a_{3}$ \\
\hline 3: & $a_{2} \quad a_{1}$ & $a_{3}$ \\
\hline & $a_{2} \sim_{4} a_{3}$ & $a_{1}$ \\
\hline
\end{tabular}
to the profile consisting of the individual rankings (of the alternatives) derived from the evaluations $\left(f_{i}(a)\right)_{a \in A}$.

From the evaluations $\left(f_{i}(a)\right)_{a \in A}$ for $i \in N$ one can derive the following profile, in which $\sim_{i}$ denotes the indifference relation of decision maker $i \in N$. 
Which alternatives will be chosen by the Plurality Rule? Since $a_{2}$ is mentioned three times as the first preference, $a_{3}$ is mentioned two times as the first preference, $a_{1}$ only once, while $a_{4}$ is the first preference of no decision maker, under the Plurality Rule the Management Committee which has to choose two alternatives, will decide for candidates $a_{2}$ and $a_{3}$. In this example, the result under the Plurality Rule coincides with the result under our procedure of consensus reaching.

When applying the Majority Rule to our example, no winner may be selected. First of all, $a_{4}$ is defeated by all remaining alternatives, alternative $a_{2}$ defeats $a_{1}, a_{1}$ defeats $a_{3}$, while $a_{2}$ does NOT defeat $a_{3}$.

Next, let us apply the Borda Rule. For equally preferred alternatives we give an average number of points. Table 5 shows the results of applying this rule.

Table 5: Applying the Borda Rule

\begin{tabular}{c|c|c|c|c|c|c}
$\begin{array}{c}i \in N \rightarrow \\
a \in A \downarrow\end{array}$ & 1 & 2 & 3 & 4 & 5 & $\sum$ \\
\hline$a_{1}$ & 3 & 3 & 3 & 1,5 & 4 & 14,5 \\
\hline$a_{2}$ & 2 & 4 & 4 & 3,5 & 1 & 14,5 \\
\hline$a_{3}$ & 4 & 2 & 2 & 3,5 & 2,5 & 14 \\
\hline$a_{4}$ & 1 & 1 & 1 & 1,5 & 2,5 & 7 \\
\hline
\end{tabular}

Hence, candidates $a_{1}$ and $a_{2}$ are chosen under the Borda Rule.

If every decision maker $i$ approves an alternative $a$ if $f_{i}(a) \geq 0.25$, then the outcome under Approval Voting is the ordering $a_{1} a_{2} a_{3}$ and the committee, faced with having to choose only two alternatives, will choose $a_{1}$ and $a_{2}$. If an alternative $a$ is approved of by agent $i$ if $f_{i}(a) \geq 0.3$, then the outcome under Approval Voting will be $a_{2}\left(a_{1} a_{3}\right)$ - where $a_{2}\left(a_{1} a_{3}\right)$ denotes that the committee is indifferent with respect to $a_{1}$ and $a_{3}$ - and the committee will choose $a_{2}$ and $a_{1}$ or $a_{2}$ and $a_{3}$, depending on the choice of the chairman.

\section{Conclusion}

In the present paper, we propose a method for a committee to choose an alternative from a set of alternatives. All alternatives are evaluated by each member of the committee with respect to given criteria, which for each agent may have different weights. The model is dynamic: the preferences of the decision makers do not have to be constant, but they may be adjusted during the process of consensus reaching, each agent taking into account arguments of the other committee members.

Our consensus approach compares favorably with the voting rules known from social choice theory, because much more detailed and sophisticated information is used, resulting in an outcome that does not depend on the applied voting rule. It also compares favorably with the game-theoretic treatments, because it is conceptually simpler and can be applied in practice more easily. 
We suggest to use the MacBeth software (www.m-macbeth.com; Bana e Costa and Vansnick, 1999; Bana e Costa et al., 2003) for determining for every agent $i$ the weights $h_{i}(c)$ of the given criteria $c$ and the values $g_{i}(c, a)$ of the different alternatives $a$ with respect to a given criterium $c$. MacBeth stands for 'Measuring Attractiveness by a Categorical Based Evaluation Technique'. Based on semantic judgements concerning the attractiveness of available alternatives, it is an interactive approach to quantify the attractiveness of each alternative, in such a way that the measurement scale constructed is an interval scale. The Macbeth software is a very user-friendly tool for measuring attractiveness of alternatives: it checks the consistency of the initial evaluations, and in case of any inconsistency it indicates to the user what is the cause of the inconsistency and how to improve on it in order to reach consistency. In Roubens et al. (2005), the MacBeth technique is applied to a certain model of coalition formation. The Macbeth software appeared to be very helpful in determining the utilities (the values) of governments to parties.

The notion of absolute judgement has also been used in Saaty's Analytical Hierarchy Process (AHP). The AHP method is described in Saaty (1977, 1980). There are fundamental differences between MacBeth and AHP. In particular, in the MacBeth approach, the absolute judgements concern differences of attractiveness, whereas in Saaty's method they concern ratios of priority, or of importance. For a critical analysis of the AHP method, see Bana e Costa and Vansnick (2001), where the authors show some inconsistencies of Saaty's method.

Topics for further research on the consensus model are incorporating the possibility of tacit coalitions among the committee members and incorporating data which may be stochastically uncertain, imprecise or colored in unknown ways.

\section{References}

[1] C.A. Bana e Costa, J.C. Vansnick, The MACBETH approach: Basic ideas, software and an application. In: N. Meskens and M. Roubens (Eds.), Advances in Decision Analysis, Kluwer Academic Publishers, Dordrecht, 1999, pp. 131-157.

[2] C.A. Bana e Costa, J.C. Vansnick, A fundamental criticism to Saaty's use of the eigenvalue procedure to derive priorities. LSE OR Working Paper 01.42, 2001. Downloadable via internet.

[3] C.A. Bana e Costa, J.M. De Corte, J.C. Vansnick, MACBETH. LSE OR Working Paper 03.56, 2003. Downloadable via internet.

[4] J.-C. de Borda, Mémoire sur les Elections au Scrutin, 1781. English translation by A. De Grazia, Mathematical Derivation of an Election System. Isis, 44 (1953) 42-51.

[5] S. J. Brams, P. C. Fishburn, Approval Voting. Birkhäuser, Boston, 1983. 
[6] S. J. Brams, P. C. Fishburn, Voting Procedures. In: Kenneth Arrow, Amartya Sen and Kotaro Suzumura (eds.), Handbook of Social Choice and Welfare. Elsevier Science, Amsterdam, 2002.

[7] S. Brams, M. A. Jones, D. M. Kilgour, Forming stable coalitions, 2003. Downloadable via internet.

[8] S. Brams, D. M. Kilgour, M. R. Sanver, A minimax procedure for electing committees, 2004. Downloadable via internet.

[9] C. Carlsson, D. Ehrenberg, P. Eklund, M. Fedrizzi, P. Gustafsson, G. Merkuryeva, T. Riissanen, A. Ventre, Consensus in distributed soft environments. European Journal of Operational Research 61 (1992) 165-185.

[10] Condorcet, Sur les Elections et autres Textes. Corpus des Oeuvres de Philosophie en Langue Francaise. Librarie Artheme Fayard, Paris, 1986.

[11] A. Laruelle, F. Valenciano, Bargaining in committees as an extension of Nash's bargaining theory. Forthcoming in the Journal of Economic Theory, 2005.

[12] A. Laruelle, F. Valenciano, Noncooperative foundations of bargaining power in committees, 2005. Downloadable via internet.

[13] H. Nurmi, Aggregation problems in policy evaluation: an overview. European Journal of Political Economy 21 (2005) 287-300.

[14] B. Peleg, Game theoretic analysis of voting in committees. Cambridge University Press, 1984.

[15] M. Roubens, A. Rusinowska, H. Swart de, Using MacBeth to determine utilities of governments to parties in coalition formation. Forthcoming in the European Journal of Operational Research, 2005.

[16] T.L. Saaty, A scaling method for priorities in hierarchical structures. Journal of Mathematical Psychology 15 (1977) 234-281.

[17] T.L. Saaty, The Analytic Hierarchy Process, McGraw-Hill, 1980.

[18] H. Swart de, A. Deemen van, E. Hout van der, P. Kop, Categoric and ordinal voting: an overview. In: Swart de et al. (Eds.), Theory and Applications of Relational Structures as Knowledge Instruments, Springer's Lecture Notes in Computer Science, LNCS 2929, Heidelberg, Germany, 2003, pp. 147-195. 